\title{
The role of lens extraction in glaucoma management
}

\author{
Jolly L. Tsui ${ }^{1,2}$, Noel C. Chan ${ }^{1,2}$, Clement C. Tham ${ }^{1,2,3}$ \\ ${ }^{1}$ Department of Ophthalmology and Visual Sciences, The Chinese University of Hong Kong, Hong Kong, China; ${ }^{2}$ Department of Ophthalmology \\ and Visual Sciences, Prince of Wales Hospital, Shatin, Hong Kong, China; ${ }^{3}$ Department of Ophthalmology and Visual Sciences, Hong Kong Eye \\ Hospital, Kowloon, Hong Kong, China \\ Contributions: (I) Conception and design: All authors; (II) Administrative support: All authors; (III) Provision of study materials or patients: All \\ authors; (IV) Collection and assembly of data: All authors; (V) Data analysis and interpretation: All authors; (VI) Manuscript writing: All authors; (VII) \\ Final approval of manuscript: All authors. \\ Correspondence to: Prof. Clement C. Tham, FCOphthHK, FHKAM. Department of Ophthalmology and Visual Sciences, The Chinese University of \\ Hong Kong, Hong Kong Eye Hospital, 147K Argyle Street, Kowloon, Hong Kong, China. Email: clemtham@cuhk.edu.hk.
}

\begin{abstract}
Cataract extraction has become a much safer procedure with the development of phacoemulsification, extending its application in ocular conditions such as glaucoma for better disease control. This review aims at summarizing the effect of lens extraction with or without combined glaucoma surgeries in different types of glaucoma and describing the intraoperative techniques and changes of glaucoma care postoperatively. A comprehensive literature search was performed through Medline and PubMed, and 67 studies were selected for this review. In primary angle closure (PAC) diseases, studies have revealed significant intraocular pressure (IOP) and medication requirement reduction after lens extraction. Fewer studies described its application in primary open angle glaucoma (POAG) and ocular hypertension (OHT), but literature available suggests that it can also lead to better disease control. Likewise, lower postoperative IOP and pressure fluctuations have been shown in normal tension glaucoma (NTG). Advanced glaucoma, shallow anterior chamber and pseudoexfoliation glaucoma (PXG) are three difficult scenarios that are commonly encountered in cataract operations. Special techniques in preventing complications such as wipe out phenomenon are depicted. Goniosynechialysis, endoscopic cyclophotocoagulation and trabecular microbypass stents are a few of the popular choices of glaucoma procedures that can be performed concomitantly with phacoemulsification but evidence of their efficacy needs to be further verified. Cataract operation can improve visual field and retinal nerve fiber layer examination parameters and it is advised to set new baselines after the surgery for subsequent progression monitoring. Clearance of an optically significant cataract results in better quality of life, and with detailed and thorough explanation of the indications, expectations and risks of the surgery, cataract extraction could be considered even in cases of advanced glaucoma.
\end{abstract}

Keywords: Cataract extraction; phacoemulsification; glaucoma; intraocular pressure (IOP)

Submitted Apr 10, 2020. Accepted for publication Jul 10, 2020.

doi: $10.21037 /$ atm-20-3251a

View this article at: http://dx.doi.org/10.21037/atm-20-3251a

\section{Introduction}

In recent years, cataract extraction has become a much safer procedure with the development of phacoemulsification and more stable irrigation systems (1). The improved safety profile has shifted the indication of the operation to also treating ocular conditions other than lenticular opacities. The role of cataract extraction in lowering the intraocular pressure (IOP) and reducing the need of IOP-lowering medications in different types of primary glaucoma has been increasingly reported, especially in those with narrow angle. This review summarizes the current evidence on the application of cataract surgery in different types of primary glaucoma, the specific surgical techniques and challenges, the variety of concomitant glaucoma surgeries, and the effect of cataract extraction on non-surgical aspects of the disease including progression monitoring and quality of life. 


\section{Lens extraction in glaucoma}

With phacoemulsification, cataract operation can now be performed through smaller corneal incisions with minimal conjunctival manipulation and intraoperative IOP fluctuations, making it safer to perform even in advanced glaucoma. The effect of lens extraction on different subtypes of primary glaucoma varies, and depending on the disease severity and control, phacoemulsification alone or combined with glaucoma surgery may be indicated. Treatment strategies related to the timing and indications of lens extraction in glaucoma are discussed below.

\section{Lens extraction in primary angle closure $(\mathrm{PAC})$ diseases}

PAC diseases refer to the occlusion of the trabecular meshwork (TM) by the peripheral iris, which is defined by the World Glaucoma Association as the inability to visualize $\geq 180^{\circ}$ of the pigmented TM on gonioscopy. It is further classified into primary angle closure suspect (PACS), PAC, and primary angle closure glaucoma (PACG) depending on the presence of IOP elevation due to aqueous outflow obstruction, and glaucomatous optic neuropathy. Angle closure disease is most commonly seen in Asians, and the prevalence varies from $0.1 \%$ to $2.5 \%$ among different ethnicities. Without treatment, $3.5 \%$ to $4.4 \%$ of PACS will progress to PAC, while $30 \%$ of PAC will progress to PACG in 5 years. To resolve the pathophysiology, treatment aims at enhancing aqueous drainage through the TM by reopening the appositionally closed parts of the angle, and there are different treatment modalities in achieving so, including laser peripheral iridotomy (LPI) with/without iridoplasty, topical medications, glaucoma surgeries, and more recently proposed, lens extraction. The ability of lens removal in opening up the closed angle lies in the elimination of a lens that is usually abnormally thick and anteriorly positioned in PAC diseases. A study (2) observing the changes in drainage angle anatomy in Chinese patients 1 year after phacoemulsification and combined phacotrabeculectomy revealed that phacoemulsification alone leads to more opening of the drainage angle and greater deepening of the anterior chamber. Lens extraction also reduces the need of further glaucoma surgery and eliminates the risk of subsequent cataract formation after an initial glaucoma procedure. With a better understanding of the anatomical and IOP-lowering effects of lens extraction, phacoemulsification alone is becoming widely accepted as an alternative surgical option to phacotrabeculectomy or trabeculectomy for PAC diseases.

For patients with PAC presenting with optically significant cataract and poorly controlled IOP, cataract extraction with or without combined trabeculectomy would be an obvious surgical option. A randomized controlled trial (RCT) (3) by Tham et al. showed that during the 24-month follow-up period, Chinese patients who underwent combined phacotrabeculectomy had lower mean IOP and required 1.25 fewer topical glaucoma medications; while in the group that had cataract extraction alone, $11.1 \%$ of the patients needed subsequent trabeculectomy within 2 years for adequate IOP control. There was no significant difference in visual acuity and glaucomatous progression between these two groups, even though this study was not designed or powered to compare glaucomatous progression. Combined phacotrabeculectomy was reported to have more postoperative complications $(26.2 \%$ vs. $8.1 \%$ in phacoemulsification alone) (4). $25 \%$ of the patients with combined phacotrabeculectomy required additional interventions, including laser suturelysis and needling to maintain filtration. Another RCT showed similar results (5). Therefore, combined phacotrabeculectomy is suggested for patients requiring greater IOP and medications reduction, especially for patients with multiple drug allergies, and poor compliance or accessibility to medications. Benefits should however be balanced against the increased risk of operative complications. For cases with low tolerance to postoperative complications related to combined phacotrabeculectomy, e.g., advanced glaucoma and lower corneal endothelial cell counts, phacoemulsification can be considered as the first line surgical option. Trabeculectomy alone has limited role in poorly controlled PAC disease with cataract as it fails to address the optically significant cataract, and may lead to further subsequent cataract progression. It is also associated with relatively higher perioperative risks and complications and the pathophysiology of PAC is not resolved.

For Chinese patients with medically controlled PAC diseases and visually significant cataract, phacoemulsification alone can similarly reduce IOP (by $9.82 \%$ ) and glaucoma medications required (by 59.2\%) (6). Combined phacotrabeculectomy can again further reduce the IOP-lowering medications by 0.8 drugs on average in a 24-month postoperative period, but is related to more complications and additional surgical interventions. So far, no association has been identified between the additional IOP reduction from combined surgery and the differences in glaucomatous progression. As phacoemulsification alone is capable of reversing the anatomically closed angles and 
preventing pupil block, thus reducing IOP, medication needs and slowing glaucomatous progression, many specialists are now more hesitant in performing combined phacotrabeculectomy in patients with optically significant cataract when the PAC diseases are successfully controlled by medications.

A follow-up retrospective analysis of the two prospective RCTs $(3,6)$ by Tham et al. described factors that correlate with IOP control failure in PACG with coexisting cataract after phacoemulsification and combined phacotrabeculectomy (7). Using multivariate analysis, the performance of phacoemulsification alone, higher preoperative IOP (odds ratio of 1.732 for every $5 \mathrm{mmHg}$ IOP increase) and prior glaucoma medication requirements are associated with incomplete success at 24 month (defined as IOP $\geq 21 \mathrm{mmHg}$ or requiring glaucoma medications to maintain an IOP $<21 \mathrm{mmHg}$, or additional glaucoma surgery to lower the IOP). Therefore, in situations as such, combined phacotrabeculectomy can be considered to achieve better IOP control, while keeping in mind the possible risk of postoperative complications.

Lens extraction as a surgical option for PAC diseases can be controversial when there is no optically significant cataract. This is sometimes referred to as "clear lens extraction'. The authors do, however, feel that this term 'clear lens extraction' is a misnomer when applied to PAC diseases, as it emphasizes possibly the only normal aspect of this lens. In most, if not all, cases of PAC diseases, this lens is pathological: it is often too thick, and/or too anteriorly positioned, and is often one important pathological mechanism underlying the angle closure. Instead of improving media clarity, this operation aims at deepening the anterior chamber and opening up the drainage angle to prevent pupil block and reduce IOP. Even without cataract, lens extraction results in a significant reduction in angle closure accompanied by an increase in anterior chamber width and depth in PAC diseases (8). Lens extraction alone may improve IOP control, with reduction in the need of glaucoma medications and surgery. Even without cataract, lens extraction alone may offer visual improvements through the correction of hypermetropia, astigmatism, and presbyopia, through the implantation of appropriate modern intraocular lenses (IOL). Modern phacoemulsification is also associated with less perioperative complications and corneal endothelial damage compared to filtration surgery. In cases where subsequent glaucoma surgeries are necessary, cataract regardless will no longer be a concern both at the point of surgery and in the future. An RCT in 2013 (9) compared the effect of phacoemulsification and trabeculectomy on Chines patients with medically uncontrolled PACG without cataract over 2 years. Both groups of eyes were found to have significant and comparable IOP reduction ( $34 \% v s$. $36 \%$ ), but trabeculectomized eyes required 1.1 fewer IOPlowering medications on average at 24 months than those who underwent phacoemulsification. Surgical complication rate is however significantly higher in trabeculectomy $(46 \%)$ than phacoemulsification $(4 \%)$, and $33 \%$ of the trabeculectomized eyes presented with cataract within 2 years after the surgery. The rate of additional surgical intervention is also higher in the trabeculectomy group, although none of the eyes remained medically uncontrolled after further procedures to maintain bleb drainage. The study concluded that both phacoemulsification and trabeculectomy are helpful in lowering IOP in medically uncontrolled PACG without visually significant cataract. Trabeculectomy can reduce IOP and medication required more effectively, but is associated with higher rate of complications. The pros, cons, and risks of these surgical options should therefore be thoroughly discussed with patients before surgery.

The role of lens extraction was further evaluated in an RCT involving 419 patients from 5 countries, all with newly diagnosed PAC and IOP of $\geq 30 \mathrm{mmHg}$ without cataract (10). Patients were randomized into receiving either lens extraction or standard care with LPI and topical medical treatment. The group that received lens extraction was found to have mean IOP $1.18 \mathrm{mmHg}$ lower than the standard care group, and fewer patients required topical IOP-lowering medications after surgery ( $21 \%$ vs. $61 \%)$. There is a much lower incidence of patient requiring additional glaucoma surgery ( $1 v s .24)$ after lens extraction, and only 1 patient (compared to 3 ) had irreversible visual loss at the end of the study. Although there is no consensus on lens extraction being a standard treatment for PAC diseases without cataract, this study has provided new evidence for its benefits and effectiveness in controlling IOP without significant increase in risks and complications. However, as a surgical procedure, phacoemulsification is not completely without risks. Early postoperative IOP spikes alone can cause wipe-out phenomenon and irreversible visual loss. The amount of aqueous outflow achieved after lens extraction is also limited by pre-existing TM damage. Indications, expectations, and potential perioperative complications of the procedures should therefore be communicated clearly and thoroughly to the patients.

For acute primary angle closure (APAC), the key 
objectives of the initial treatment are to lower IOP, reduce symptoms, and to prevent further ocular tissue injuries. This can be achieved by IOP-lowering medications, laser peripheral iridoplasty, anterior chamber paracentesis, and/or corneal indentation. After aborting the attack, conventional therapy has been LPI, yet more studies are now indicating the benefits of early phacoemulsification instead. An RCT (11) performed by Lam et al. has shown that in Chinese patients, there is a significantly higher risk of IOP >21 $\mathrm{mmHg}$ after LPI alone, while early phacoemulsification is only associated with $3.2 \%$ IOP rise and the mean IOP is consistently lower at all time points during the 1.5 -year study period. The mean number of medications required is also considerably higher after LPI as compared to early phacoemulsification ( 0.9 vs. 0.03$)$. Another RCT involving mostly Chinese patients (12) compared the 2-year efficacy of phacoemulsification and LPI performed within 1 week after APAC and they revealed higher rate of successful IOP control in phacoemulsification (89.5\%) than LPI (61.1\%). Flushing of the TM and the wider opening of the drainage angle after phacoemulsification are hypothesized to have led to higher aqueous outflow and thus lower IOP. While phacoemulsification is becoming more recognised as an initial treatment of APAC, the optimal timing to perform the surgery is still debatable. Phacoemulsification performed as early as a few days after abortion of the acute attack might prevent the formation of peripheral anterior synechiae (PAS), but is associated with much higher surgical risk due to corneal haze and still active inflammation. If surgery is performed at a later stage, i.e., weeks after corneal oedema and inflammation subsides, the surgical risk is much reduced but PAS is likely to have set in. Currently, the authors believe that the best time window for safely performing phacoemulsification in post-APAC eyes for maximum angle opening may be when the inflammation has just largely settled. This time window may vary from patient to patient, and is likely to be around 2 to 3 weeks after the initial APAC attack, depending on the severity of the associated inflammation. While patients await lens extraction, LPI may be considered to reduce interval attack, but care should be taken to minimize the induction of more inflammation, or further damage to the corneal endothelium.

\section{Lens extraction in primary open angle glaucoma (POAG) and ocular bypertension (OHT)}

POAG is the most common type of primary glaucoma with a prevalence of $3.05 \%$ worldwide, affecting 44.11 million in 2013 , and the number is expected to rise to 52.68 and 79.76 million in 2020 and 2040, respectively (13). As the lens is less likely to have a major direct mechanistic role in the disease pathogenesis, cataract extraction is less well studied in POAG compared to PAC diseases. However, cataract surgery has also been shown to reduce IOP and medication requirement in POAG. A comparative case series (14) in 2012 randomized 42 patients from the Ocular Hypertension Treatment Study into either phacoemulsification group or control group. After cataract extraction, the mean postoperative IOP was $16.5 \%$ lower than the preoperative values, and $39.7 \%$ of eyes had $\geq 20 \%$ reduction in postoperative IOP, which remained lower for at least 36 months. Higher preoperative IOPs were associated with greater reduction after the operation. These findings were also confirmed by a review published in 2012 that demonstrated sustained IOP reduction of $1.5 \mathrm{mmHg}$ in eyes with OAG after phacoemulsification (15). Another prospective study performed in the United States revealed that narrower preoperative angles result in greater IOP drop. Preoperative IOP, angle opening distance, and lens vault are predictors for IOP reduction (16). Nonetheless, the decrease in IOP observed after phacoemulsification in glaucoma with open angles is not as pronounced as in PAC diseases. In situations where greater IOP reduction is targeted, studies have suggested performing combined phacotrabeculectomy for fewer early postoperative IOP spikes and IOP-lowering medications needed (17). In advanced open angle glaucoma with visually significant cataract, phacoemulsification and combined phacotrabeculectomy can both result in IOP reduction, but combined procedure leads to greater decrease in IOP $(1.7 \mathrm{mmHg})$ and number of glaucoma medications required (0.6 medications) (18). Visual acuity improvement is similar between the two groups, but eyes that underwent phacoemulsification alone experienced more IOP spikes over $25 \mathrm{mmHg}$ in the early postoperative period. Although cataract operation is less well recognised as an IOPlowering surgery in POAG, it can be considered in eyes with visually significant cataract for its additional IOPlowering effect. It also prevents cataract formation in cases where further glaucoma surgeries are likely necessary in the future. Combined phacotrabeculectomy is associated with more complications and less IOP-lowering effect compared to phacoemulsification followed by trabeculectomy alone, and phacoemulsification in trabeculectomized eyes has been to found to increase filtration failure rate (19), leading to 
an average of $2 \mathrm{mmHg}$ rise in IOP after 12 months (15). Earlier cataract extraction precludes such risks.

\section{Lens extraction in normal tension glaucoma (NTG)}

NTG, being considered as a continuum/variant of POAG, is characterized by glaucomatous optic neuropathy in the presence of open anterior chamber angle and IOP $\leq 21 \mathrm{mmHg}$ not otherwise accountable by other diseases. With a normal IOP, IOP-independent factors are believed to play an important role in the pathogenesis and disease progression in this subtype of glaucoma. Ocular blood flow (OBF) instability is one of the most discussed vascular pathogenic mechanisms (20), and it is closely related to the ocular perfusion pressure, which is the difference between the arterial blood pressure and IOP. OBF instability can be a result of IOP fluctuations beyond normal autoregulation, or normal IOP and/or blood pressure fluctuations in the background of altered autoregulation. IOP evaluation in NTG through contact lens sensor has revealed $80 \%$ nocturnal acrophase and prolonged IOP elevations (21), and high IOP fluctuations with peaks $>90 \mathrm{mV}$ have been linked to glaucomatous progression $(22,23)$. A prospective open-label study performed in Japan (24) examined the IOP fluctuation of NTG eyes before and after cataract surgery with contact lens sensors. It was found that the mean IOP was significantly reduced from preoperative IOP of 14.7 to $11.4 \mathrm{mmHg}$. Postoperative IOP fluctuation over 24 hours is also significantly lower than their preoperative equivalents. Another prospective observational study conducted in Korea confirmed the reduction in IOP, and further demonstrated an increase in angle parameters that is linearly correlated with postoperative IOP changes in eyes with NTG compared to control group (25). Current evidence suggests that lens extraction can be considered in NTG eyes with optically significant cataract for its additional benefits of IOP reduction and stabilization. Whether it should be performed as a standard management of NTG, especially in patients without visually significant cataract, will need verification from further clinical trials.

\section{Pearls in performing phacoemulsification in different types and severity of glaucoma}

Surgical challenges may arise when extracting cataract in glaucoma eyes, due to the unique anatomy. Special considerations and precautions must be taken, especially when operating on eyes with advanced glaucoma. Before the surgery, thorough and detailed explanation should be given on the risks, particularly those specific to the conditions such as wipe out phenomenon in advanced glaucoma and corneal decompensation in low corneal endothelial cell counts. The risk of subsequent failure of filtration in trabeculectomized eyes, and the expectations on IOP-lowering and visual outcomes, should be thoroughly discussed with patients. Options of combined glaucoma surgery for further IOP reduction should be mentioned. The surgery should be carefully planned to minimize the risk of further damage to eyes already compromised by preexisting glaucoma, and taking measures, e.g., minimizing conjunctival manipulations, in case future glaucoma surgeries are needed.

Apart from the complications general to lens extraction in all eyes, patients with PAC have heightened risk of malignant glaucoma postoperatively, which can potentially cause further damage to the residual retinal nerve fibre function if not treated timely and adequately. Artificial intraocular lens insertion can also lead to secondary glaucoma such as pigment dispersion syndrome and uveitisglaucoma-hyphaema syndrome from the chafing effect of the haptics on the posterior iris pigment epithelium, steroid-induced glaucoma from the use of steroid eye drops postoperatively, and secondary angle closure related to the development of Soemmering's rings. Lens extraction may also induce flaring of uveitic glaucoma and progression of ocular ischaemia in the form of neovascular glaucoma in the presence of pre-existing uveitis history and ischaemic retinopathies respectively. In the rare occurrence of epithelial downgrowth from surgical wounds, refractory glaucoma can occur together with other debilitating complications, e.g., retro-corneal membrane and corneal oedema. Aphakic glaucoma may also ensue if intraocular lenses are not inserted. Compared to native lenses, intraocular lenses lack the ability to accommodate, which is a particularly important consideration for younger patients. It is crucial to explain to patients that, although lens extraction may be effective in reducing IOP and IOPlowering medication requirements particularly in PAC diseases, it can lead to other types of glaucoma, and the loss of accommodation and blinding complications should be taken into full consideration.

\section{Advanced glaucoma}

Eyes with advanced glaucoma are at risk of wipe out phenomenon, which is believed to be due to IOP 
fluctuations and spikes intra- and post-operatively. Before surgery, IOP should be adequately controlled with topical and/or systemic medical treatment, like acetazolamide and mannitol. During the operation, minimal bottle height while maintaining a stable anterior chamber for cataract extraction should be employed. IOP fluctuations should also be avoided by minimizing the collapse of anterior chamber during insertion and removal of instruments. Every effort should be taken to prevent IOP spikes by ensuring thorough removal of the ophthalmic viscosurgical devices (OVDs), and initiating topical glaucoma medications or even short-term oral acetazolamide early to avoid wipe out phenomenon. More frequent follow-up should be considered to closely monitor the postoperative IOP.

\section{Shallow anterior chamber}

In eyes with shallow anterior chamber, especially those with previous APAC attack and anterior chamber laser procedures, corneal endothelial cell count should be evaluated using specular microscopy so that patients can be appropriately counselled prior to surgery. Special manoeuvres can be taken intraoperatively to preserve remaining endothelial function and prevent corneal oedema postoperatively. Combined phacoemulsification and endothelial keratoplasty should be discussed if the endothelial cell status is unlikely to be able to withstand the stress and potential damage induced by cataract surgery. To maintain a stable anterior chamber and prevent iris prolapse, it is important to ensure a secure main wound using a 3-step-incision and paracentesis wounds should be tight angled. Main wound can also be created after capsulorhexis is completed through a paracentesis wound to ensure anterior chamber stability. Specialized heavy OVDs, such as Healon GV/5, can be used to deepen the anterior chamber, prevent iris movement, and tamponade the anterior bulging curvature of the lens during capsulorhexis. Apart from using heavier OVDs, it is crucial to maintain a centripetal force while performing capsulorhexis, and a second instrument can also be used to depress the lens. Excessive hydrodissection should be avoided to prevent iris prolapse and inadvertent posterior capsular blowout with subsequent dropped nucleus. Bottle height should be elevated to maintain adequate pressure in the anterior chamber during the whole procedure to allow easier and safer manipulation, and avoid endothelial damage. OVDs, especially heavy ones, should be removed thoroughly, and glaucoma medications should be prescribed to minimize
IOP rise postoperatively. Eyes with PAC disease are predisposed to increased risk of malignant glaucoma, which needs to be managed with immediate cycloplegia and YAG laser hyaloidotomy. In refractory cases, vitrectomy combined with iridectomy, hyaloidectomy, and zonulectomy need be considered.

\section{Pseudoexfoliation glaucoma (PXG)}

PXG is a secondary open-angle glaucoma commonly affecting one eye, and it is associated with an age-related systemic disorder [pseudoexfoliation syndrome (PXF)] where fibrillar proteinaceous substance is produced in abnormally high concentration and deposited onto the ocular tissues including the TM, causing obstruction to aqueous outflow. Cataract develops earlier and more often in PXF. Pseudoexfoliative materials deposition, poorly dilated pupils, fragile zonules, and low endothelial cell count make cataract surgery more challenging (26). Before surgery, these risk factors should be evaluated for better surgical planning and preoperative counselling.

Apart from routine preoperative dilating eye drops, any miotics should be stopped for at least a week before the surgery. Preoperative topical non-steroidal antiinflammatory drug may be considered. Posterior synechiae should be lysed with OVDs or capsulorhexis forceps after stabilizing the anterior chamber with OVDs. The pupil can then be dilated with cohesive OVDs, or pupil stretching and expansion devise, e.g., iris hooks or pupillary rings (27). Iris hooks can also provide additional support to the anterior capsulotomy in cases of zonular weakness. Adequate hydrodelineation of the cataract should be performed so that the lens can be debulked and removed by layers to minimize stress to the zonules. Intracameral miotics can be used at the end of the surgery to reverse pupil dilation and prevent immediate PAS formation. More intensive topical steroid eye drops are required after the surgery to control the ocular inflammation from iris manipulation and prevent cystoid macular oedema. Postoperative topical non-steroidal anti-inflammatory drugs can also reduce the amount of aqueous protein release and the percentage of retinal thickening in PXF (28).

The presence of zonular weakness may make capsulorhexis difficult due to the lack of anterior capsular tension. Sharp instruments are therefore needed to make the first puncture on the anterior capsule, and instruments such as iris hook can be used to exert countertraction on the torn edges of the anterior capsule to complete the 
capsulorhexis. Trypan blue can be used to stain the anterior capsule when deposition of pseudoexfoliation materials makes visualization difficult $(29,30)$. The size of the capsulorhexis should be sufficient to allow zonule-friendly phacoemulsification, but not too large that the insertion of capsular stabilization devices becomes impossible. The choice of capsular stabilization devices depends on the severity of zonular instability. When the weakness is mild, capsular tension rings (CTR) can be considered to support intraocular manoeuvres and stabilize intraocular lens implantation in the capsular bag. For more serious zonular weakness, Cionni modified-CTR (mCTR), or capsular tension segment (CTS) can be used, as both of which can be sutured onto the sclera for further stabilization and fixation. Moreover, CTS does not require dialling motion and so minimizes stress on the zonules. Some surgeons even suggested a routine use of CTR in PXF for easier surgery and long term IOL stability in view of possible progressive zonular weakening (31). To aid cortex removal after CTR insertion, OVDs can be used for hydrodissection to create a greater cleavage. Horizontal or vertical chopping techniques can minimize stress on the zonules during nucleus removal, and a tangential stripping combined with centripetal movements should be utilized to retrieve the cortex especially that trapped behind the CTR (32). Dry removal with a cannula can even be considered in difficult situations. In cases of zonular instability, one-piece acrylic intraocular lens together with CTR can be considered to allow better centration with minimal capsular and zonular stress.

Endothelial cells are often compromised in PXF, resulting in more corneal oedema or even decompensation after cataract surgery. The potential risks and additional surgeries implicated in these cases should be thoroughly discussed. To protect the endothelium during phacoemulsification and intraocular lens implantation, soft-shell technique can be applied (33). It involves the injection of dispersive OVDs such as Viscoat close to the endothelium, and then cohesive OVDs such as Healon or Provisc underneath to spread out the dispersive OVDs evenly on the endothelial surface, creating a protective 'shell' coating from ultrasonic energy and manipulation in the anterior chamber. Care must be taken to remove all the OVDs from the anterior chamber at the end of the operation to prevent IOP spikes. Postoperative IOP-lowering medications should be given in advanced glaucoma and when removal of OVDs could be incomplete.

\section{Adjunctive procedures performed alongside phacoemulsification}

For further IOP-lowering effect, phacoemulsification can be combined with other procedures. Studies verifying the efficacy and safety of combined procedures are still emerging. Our review will mainly focus on goniosynechialysis (GSL), endoscopic cyclophotocoagulation, and trabecular microbypass stents.

\section{Goniosynechialysis (GSL)}

GSL involves the mechanical disruption of PAS, and can be combined with lens surgery. This procedure involves mechanically stripping the PAS away from the drainage angle, re-exposing the TM to improve aqueous outflow, with the assumption that the TM and the downstream physiological drainage pathway have remained functional. It can be performed under direct visualization of the anterior chamber angles with either endoscopic instruments (34), or goniolenses such as Koeppe/Barkan lens with a $45^{\circ}$-tilted operating microscope. The peripheral iris near the PAS is pushed down with a blunt spatula, or dense OVDs, or gently pulled away towards the centre of the pupil until the TM or scleral spurs are exposed. Care should be taken not to damage the iris or induce iridodialysis. Intracameral miotics can keep the iridocorneal angle open, while air injected into anterior chamber can reduce postoperative bleeding from the iris and angle after the procedure. Postoperatively, miotics can be prescribed to prevent PAS reformation. The advantages of performing GSL together with phacoemulsification is that it allows better visualization and access to the angle without making another incision. It also eliminates the risk of lens damage and cataract formation. However, this procedure has risk of intraoperative haemorrhage, iridodialysis and cyclodialysis. So far, studies have been inconsistent in showing additional IOP-lowering effect from combined phaco-GSL, compared to phacoemulsification alone (35). Reduction of antiglaucoma eye drops use is also not proven. Further studies involving more subjects and longer follow-up with more standardized surgical techniques including the extent of GSL are required to provide more data on the true efficacy of GSL. The timing of performing GSL after APAC attack may also affect the efficacy in IOP reduction. For GSL that is performed within 6 months after the acute attack, study has reported $90.4 \%$ of the eyes having IOP $<20 \mathrm{mmHg}$ 
without medications (36). A positive correlation between the attack-to-surgery time interval and postoperative IOP was described (37). It is believed that long standing PAS are associated with irreversible pathological changes in the TM, which ultimately determines the amount of aqueous outflow along the drainage pathway. One should look out for wipe out phenomenon due to significant IOP spikes occurring in advanced glaucoma during and immediately after GSL.

\section{Endoscopic cyclophotocoagulation (ECP)}

ECP is the ablation of the ciliary body intraocularly through an endoscopic fiberoptic probe to suppress aqueous humor production, and thereby lowering IOP. It can also be used as an endocycloplasty to treat plateau iris by inducing shrinkage and retraction of the ciliary process and iris roots (38). Combining this procedure with phacoemulsification allows direct visualization of the treatment site without additional incisional wounds and reduces the collateral tissue damage, especially when anterior segment anatomy is distorted, as compared to conventional transscleral cyclophotocoagulation. A review published in 2018 summarized the current evidence on the efficacy and safety of phaco-ECP (39). All the seven included retrospective studies demonstrated decreased postoperative IOP after phaco-ECP. The majority of them revealed a reduction in IOP-lowering medications needed after the surgery. Higher success rate was noted in higher preoperative IOP (40) and POAG eyes (41). Francis et al. reported in their prospective study that (42) postoperative IOP in medically controlled OAG was lowered from 18.1 to $16 \mathrm{mmHg}$, and the mean number of glaucoma medications used decreased from 1.5 to 0.4 at 2 years after phaco-ECP. Phacoemulsification alone showed no significant changes in mean IOP and number of glaucoma medications pre- and postoperatively. Another prospective study published earlier also concluded that phaco-ECP was an effective alternative to phaco-trabeculectomy in reducing IOP and medication requirement, and was reasonably safe with a similar rate of additional surgical intervention (43). Compared to trabeculectomy alone, ECP is generally associated with less serious complications except in refractory glaucoma (44), and the most common complications are IOP spikes $(14.5 \%)$ and hemorrhages $(3.8 \%)$ according to the ECP Collaborative Study (45). Performing ECP with phacoemulsification has not been shown to increase the risk of serious complications compared to phacoemulsification alone (42). It should however be noted that ECP can alter the postoperative lens position and lead to more myopic refractive outcomes $(46,47)$. Adjustment should be made in the selection of the appropriate lens power. Nonetheless, most of the studies available now are performed in Caucasians and retrospective in design, without specifying the type of glaucoma included, and the treatment extent and settings applied. Evidence for the long-term effect of ECP beyond 36 months is also lacking. A prospective randomized controlled trial comparing the effect of phacoemulsification alone and phaco-ECP in PACG is now underway. More studies as such are needed to establish the medium and long-term effect of phaco-ECP in POAG and PAC diseases.

\section{Trabecular microbypass stents}

Together with phacoemulsification, various stents have been developed to bypass or enhance the natural outflow pathways, and one or more of these stents can be injected into the anterior chamber angle using the same incision wound for cataract extraction. Two of the more commonly available stents are the iStent (Glaukos, Laguna Hills, CA, USA) and the Hydrus Microstent (Ivantis Inc., Irvine, CA, USA). iStents are heparin-coated titanium implants that can be placed in the TM through an injector. A systemic review published in 2019 included 7 RCTs that evaluated iStent use in OAG (48), but the results showed that there was only very low-quality evidence that such device can lead to better IOP or eliminate the use of glaucoma medications in the short, medium, and long term. The RCTs available up till this day are all funded by Glaukos with more than half of the studies demonstrating questionable randomization methods and study design. Results from thirteen studies yet to be published might provide more data on the benefit of iStents. Hydrus Microstents is made of nickeltitanium alloy, and it acts as a dilator of the Schlemm's canal to enhance aqueous outflow. Two RCTs compared phacoemulsification alone $v s$. combined phaco-microstent and they demonstrated that combined surgery in mild to moderate POAG had superior reduction in modified diurnal IOP and medication use at 2 years $(49,50)$. However, these are sponsored studies and the long-term safety and efficacy of these microstents will need to be further verified.

\section{The effect of lens extraction on visual field (VF) and retinal nerve fibre layer (RNFL) monitoring}

The effect of lens extraction on VF and RNFL examination interpretation has been evaluated by numerous studies. 
It was shown that cataract surgery is related to significant mean deviation (MD) improvement postoperatively (51-53). Some studies also revealed similar or slight deterioration in pattern standard deviation (PSD) (54-56). Pre- and postoperative visual field index (VFI) were found to be similar (57), except in mild glaucoma of $\mathrm{MD}>-6 \mathrm{~dB}$ and the presence of cortical cataract (58), making it possibly a more robust measure of VF damage than MD and PSD in glaucomatous eyes with coexisting cataract (59). Changes in $\mathrm{MD}$ were especially related to posterior subcapsular cataract $(52,57)$, while VF parameters are generally less affected by nuclear sclerosis (58). Glaucoma progression index also has statistically significant improvement after cataract extraction (60). However, most of these studies are retrospective in design, and more evidence from prospective controlled clinical trials is required to confirm these findings. Similarly, cataract extraction may result in better sensitivity in RNFL examination. Studies have shown significant increase in RNFL thickness and signal strength using spectral-domain and time-domain optical coherence tomography $(61,62)$ and scanning laser polarimetry $(63)$, especially over the inferior quadrant, which is usually the first area of RNFL thinning detected in glaucoma. Ganglion cell complex thickness measurement also increased after cataract removal (64). Like VF, posterior subcapsular cataract has the most interference of the results (65). Due to effect of lenticular opacities on examination results, VF and RNFL assessment in glaucoma patients with cataract should be interpreted carefully and new baselines are recommended for future progression monitoring after cataract extraction.

\section{Cataract extraction and quality of life in patients with glaucoma}

Although no reviews and RCTs have been conducted yet, various studies have demonstrated that elimination of visually significant cataract can lead to improved visual function and vision-related quality of life despite preexisting visual field defects $(52,55,66)$. For patients with advanced glaucoma where there is near total cupping of the optic nerve and severe visual field loss within $10^{\circ}$ of fixation, significant increase in the total and subscales of the Chinese version of the Low Vision Quality of Life Questionnaire (CLVQOL) was noted after cataract extraction. Greater improvement in the composite scores was associated with better preoperative weighted average LogMAR and age below 60 years (67). Even in patients with no optically significant cataract, lens extraction in PACG could help maintain good visual acuity and improve visual function and quality of life as measured by the European Quality of Life5 Dimensions questionnaire, possibly due to the correction of hypermetropic refractive error, which is often found in patients with PAC disease (10). Therefore, for the benefit of improving visual functioning and quality of life, option of cataract extraction should be discussed with patients with glaucoma, explaining in detail the related risks and implications.

\section{Points to note}

(I) Lens extraction can reduce IOP and IOP-lowering medication requirements in PAC disease. However, associated risks should be considered and carefully communicated to patients;

(II) In PAC diseases with no optically significant cataract, clear lens extraction has been advocated for its superior ability in reducing IOP and IOP-lowering medication requirements compared to the standard treatment with LPI and medical therapy, and for its additional benefit of improving visual function by correcting refractive errors;

(III) Combined phacotrabeculectomy can lead to further reduction in IOP and IOP-lowering medication requirements in PAC diseases but is related to increased risk of complications;

(IV) The effect of lens extraction in POAG and OHT is less well studied but literature has suggested its effectiveness in reducing IOP and IOP-lowering medication requirements even though the results are not as pronounced compared to PAC disease;

(V) Combined phacotrabeculectomy in POAG and OHT can result in further IOP and IOP-lowering medication requirement reduction compared to lens extraction alone but is associated with more complications;

(VI) Earlier lens extraction has the benefit of preventing cataract formation in cases where further glaucoma surgeries are required and filtration failure in those previously trabeculectomized;

(VII) Lens extraction has been shown to be able to reduce the postoperative IOP and diurnal IOP variations in NTG, which has been proposed to be an important pathogenic mechanism in this specific glaucoma subtype. 


\section{Conclusions}

Lens extraction has become a much safer procedure and is more commonly performed in various types of glaucoma, especially in PAC diseases, as a part of the glaucoma management. For patients requiring more significant reduction in IOP, combined glaucoma surgery can be offered after the consideration of increased risks and complications. Special techniques and precautions need to be applied to ensure safe and uncomplicated cataract operation. Different minimally invasive glaucoma procedures can also be combined with phacoemulsification for additional IOPlowering, but their long-term efficacy awaits confirmation by future studies. Apart from improving the IOP control, lens extraction also alters the sensitivity of visual field and retinal nerve fibre layer examination, and new baselines should be established for future comparison. Like cataract operation in normal individuals, cataract operation can lead to improved visual functioning and quality of life in glaucoma patients.

\section{Acknowledgments}

Funding: None.

\section{Footnotes}

Provenance and Peer Review: This article was commissioned by the Guest Editor (Dr. Andrzej Grzybowski) for the series "Recent developments in cataract surgery" published in Annals of Translational Medicine. The article was sent for external peer review organized by the Guest Editor and the editorial office.

Peer Review File: Available at http://dx.doi.org/10.21037/ atm-20-3251a

Conflicts of Interest: All authors have completed the ICMJE uniform disclosure form (available at http:// dx.doi.org/10.21037/atm-20-3251a). The series "Recent developments in cataract surgery" was commissioned by the editorial office without any funding or sponsorship. The authors have no other conflicts of interest to declare.

Ethical Statement: The authors are accountable for all aspects of the work in ensuring that questions related to the accuracy or integrity of any part of the work are appropriately investigated and resolved.

Open Access Statement: This is an Open Access article distributed in accordance with the Creative Commons Attribution-NonCommercial-NoDerivs 4.0 International License (CC BY-NC-ND 4.0), which permits the noncommercial replication and distribution of the article with the strict proviso that no changes or edits are made and the original work is properly cited (including links to both the formal publication through the relevant DOI and the license). See: https://creativecommons.org/licenses/by-nc-nd/4.0/.

\section{References}

1. Davis G. The Evolution of Cataract Surgery. Mo Med 2016;113:58-62.

2. Tham CC, Leung DY, Kwong YY, et al. Effects of phacoemulsification vs. combined phaco-trabeculectomy on drainage angle status in primary angle closure glaucoma (PACG). J Glaucoma 2010;19:119-23.

3. Tham CC, Kwong YY, Leung DY, et al. Phacoemulsification vs. combined phacotrabeculectomy in medically uncontrolled chronic angle closure glaucoma with cataracts. Ophthalmology 2009;116:725-31, 31.e1-3.

4. Tham CC, Kwong YY, Leung DY, et al.

Phacoemulsification vs. phacotrabeculectomy in chronic angle-closure glaucoma with cataract: complications. Arch Ophthalmol 2010;128:303-11.

5. El Sayed YM, Elhusseiny AM, Albalkini AS, et al. Mitomycin C-augmented Phacotrabeculectomy Vs. Phacoemulsification in Primary Angle-closure Glaucoma: A Randomized Controlled Study. J Glaucoma 2019;28:911-5.

6. Tham CC, Kwong YY, Leung DY, et al. Phacoemulsification vs. combined phacotrabeculectomy in medically controlled chronic angle closure glaucoma with cataract. Ophthalmology 2008;115:2167-73.e2.

7. Tham CC, Leung DY, Kwong YY, et al. Factors correlating with failure to control intraocular pressure in primary angle-closure glaucoma eyes with coexisting cataract treated by phacoemulsification or combined phacotrabeculectomy. Asia Pac J Ophthalmol (Phila) 2015;4:56-9.

8. Man X, Chan NC, Baig N, et al. Anatomical effects of clear lens extraction by phacoemulsification vs. trabeculectomy on anterior chamber drainage angle in primary angle-closure glaucoma (PACG) patients. Graefes Arch Clin Exp Ophthalmol 2015;253:773-8.

9. Tham CC, Kwong YY, Baig N, et al. Phacoemulsification vs. trabeculectomy in medically uncontrolled chronic angle-closure glaucoma without cataract. Ophthalmology 
2013;120:62-7.

10. Azuara-Blanco A, Burr J, Ramsay C, et al. Effectiveness of early lens extraction for the treatment of primary angleclosure glaucoma (EAGLE): a randomised controlled trial. Lancet 2016;388:1389-97.

11. Lam DS, Leung DY, Tham CC, et al. Randomized trial of early phacoemulsification vs. peripheral iridotomy to prevent intraocular pressure rise after acute primary angle closure. Ophthalmology 2008;115:1134-40.

12. Husain R, Gazzard G, Aung T, et al. Initial management of acute primary angle closure: a randomized trial comparing phacoemulsification with laser peripheral iridotomy. Ophthalmology 2012;119:2274-81.

13. Tham YC, Li X, Wong TY, et al. Global prevalence of glaucoma and projections of glaucoma burden through 2040: a systematic review and meta-analysis. Ophthalmology 2014;121:2081-90.

14. Mansberger SL, Gordon MO, Jampel H, et al. Reduction in intraocular pressure after cataract extraction: the Ocular Hypertension Treatment Study. Ophthalmology 2012;119:1826-31.

15. Augustinus CJ, Zeyen T. The effect of phacoemulsification and combined phaco/glaucoma procedures on the intraocular pressure in open-angle glaucoma. A review of the literature. Bull Soc Belge Ophtalmol 2012:51-66.

16. Hsia YC, Moghimi S, Coh P, et al. Anterior segment parameters as predictors of intraocular pressure reduction after phacoemulsification in eyes with open-angle glaucoma. J Cataract Refract Surg 2017;43:879-85.

17. Storr-Paulsen A, Pedersen JH, Laugesen C. A prospective study of combined phacoemulsification-trabeculectomy vs. conventional phacoemulsification in cataract patients with coexisting open angle glaucoma. Acta Ophthalmol Scand 1998;76:696-9.

18. Liaska A, Papaconstantinou D, Georgalas I, et al. Phacotrabeculectomy in controlled, advanced, open-angle glaucoma and cataract: parallel, randomized clinical study of efficacy and safety. Semin Ophthalmol 2014;29:226-35.

19. Husain R, Liang S, Foster PJ, et al. Cataract surgery after trabeculectomy: the effect on trabeculectomy function. Arch Ophthalmol 2012;130:165-70.

20. Trivli A, Koliarakis I, Terzidou C, et al. Normal-tension glaucoma: Pathogenesis and genetics. Exp Ther Med 2019;17:563-74.

21. Agnifili L, Mastropasqua R, Frezzotti P, et al. Circadian intraocular pressure patterns in healthy subjects, primary open angle and normal tension glaucoma patients with a contact lens sensor. Acta Ophthalmol 2015;93:e14-21.
22. De Moraes CG, Jasien JV, Simon-Zoula S, et al. Visual Field Change and 24-Hour IOP-Related Profile with a Contact Lens Sensor in Treated Glaucoma Patients. Ophthalmology 2016;123:744-53.

23. Choi J, Kook MS. Systemic and Ocular Hemodynamic Risk Factors in Glaucoma. Biomed Res Int 2015;2015:141905.

24. Tojo N, Otsuka M, Hayashi A. Comparison of intraocular pressure fluctuation before and after cataract surgeries in normal-tension glaucoma patients. Eur J Ophthalmol 2019;29:516-23.

25. Lee W, Bae HW, Kim CY, et al. The change of anterior segment parameters after cataract surgery in normaltension glaucoma. Int J Ophthalmol 2017;10:1239-45.

26. Fontana L, Coassin M, Iovieno A, et al. Cataract surgery in patients with pseudoex-foliation syndrome: current updates. Clin Ophthalmol 2017;11:1377-83.

27. Hashemi H, Seyedian MA, Mohammadpour M. Small pupil and cataract surgery. Curr Opin Ophthalmol 2015;26:3-9.

28. Coassin M, Iovieno A, Soldani A, et al. Bromfenac ophthalmic solution $0.09 \%$ as an adjunctive therapy to topical steroids after cataract surgery in pseudoexfoliation syndrome. J Cataract Refract Surg 2016;42:1119-25.

29. Wollensak G, Wollensak J. Double contour of the lens capsule edges after continuous curvilinear capsulorhexis. Graefes Arch Clin Exp Ophthalmol 1997;235:204-7.

30. Greenburg DL, Coan EB. Capsule-splitting phenomenon during capsulorhexis in pseudoexfoliation. J Cataract Refract Surg 2015;41:1306-9.

31. Bayraktar S, Altan T, Kucuksumer Y, et al. Capsular tension ring implantation after capsulorhexis in phacoemulsification of cataracts associated with pseudoexfoliation syndrome. Intraoperative complications and early postoperative findings. J Cataract Refract Surg 2001;27:1620-8.

32. Mansour AM, Antonios RS, Ahmed, II. Central cortical cleanup and zonular deficiency. Clin Ophthalmol 2016;10:1919-23.

33. Arshinoff SA. Dispersive-cohesive viscoelastic soft shell technique. J Cataract Refract Surg 1999;25:167-73.

34. Nie L, Pan W, Fang A, et al. Combined Phacoemulsification and Goniosynechialysis under an Endoscope for Chronic Primary Angle-Closure Glaucoma. J Ophthalmol 2018;2018:8160184.

35. Wang N, Jia SB. Phacoemulsification with or without goniosynechialysis for angle-closure glaucoma: a global Meta-analysis based on randomized controlled trials. Int J 
Ophthalmol 2019;12:826-33.

36. Teekhasaenee C, Ritch R. Combined phacoemulsification and goniosynechialysis for uncontrolled chronic angleclosure glaucoma after acute angle-closure glaucoma. Ophthalmology 1999;106:669-74; discussion 674-5.

37. Fakhraie G, Vahedian Z, Moghimi S, et al. Phacoemulsification and goniosynechialysis for the management of refractory acute angle closure. Eur J Ophthalmol 2012;22:714-8.

38. Hollander DA, Pennesi ME, Alvarado JA. Management of plateau iris syndrome with cataract extraction and endoscopic cyclophotocoagulation. Exp Eye Res 2017;158:190-4.

39. Sun W, Yu CY, Tong JP. A review of combined phacoemulsification and endoscopic cyclophotocoagulation: efficacy and safety. Int J Ophthalmol 2018;11:1396-402.

40. Roberts SJ, Mulvahill M, SooHoo JR, et al. Efficacy of combined cataract extraction and endoscopic cyclophotocoagulation for the reduction of intraocular pressure and medication burden. Int J Ophthalmol 2016;9:693-8.

41. Morales J, Al Qahtani M, Khandekar R, et al. Intraocular Pressure Following Phacoemulsification and Endoscopic Cyclophotocoagulation for Advanced Glaucoma: 1-Year Outcomes. J Glaucoma 2015;24:e157-62.

42. Francis BA, Berke SJ, Dustin L, et al. Endoscopic cyclophotocoagulation combined with phacoemulsification vs. phacoemulsification alone in medically controlled glaucoma. J Cataract Refract Surg 2014;40:1313-21.

43. Gayton JL, Van Der Karr M, Sanders V. Combined cataract and glaucoma surgery: trabeculectomy vs. endoscopic laser cycloablation. J Cataract Refract Surg 1999;25:1214-9.

44. Ishida K. Update on results and complications of cyclophotocoagulation. Curr Opin Ophthalmol 2013;24:102-10.

45. Marco S, Damji KF, Nazarali S, et al. Cataract and Glaucoma Surgery: Endoscopic Cyclophotocoagulation vs. Trabeculectomy. Middle East Afr J Ophthalmol 2017;24:177-82.

46. Wang JC, Campos-Moller X, Shah M, et al. Effect of endocyclophotocoagulation on refractive outcomes in angle-closure eyes after phacoemulsification and posterior chamber intraocular lens implantation. J Cataract Refract Surg 2016;42:132-7.

47. Sheybani A, Saboori M, Kim JM, et al. Effect of endoscopic cyclophotocoagulation on refractive outcomes when combined with cataract surgery. Can J Ophthalmol 2015;50:197-201.

48. Le JT, Bicket AK, Wang L, et al. Ab interno trabecular bypass surgery with iStent for open-angle glaucoma. Cochrane Database Syst Rev 2019;3:CD012743.

49. Pfeiffer N, Garcia-Feijoo J, Martinez-de-la-Casa JM, et al. A Randomized Trial of a Schlemm's Canal Microstent with Phacoemulsification for Reducing Intraocular Pressure in Open-Angle Glaucoma. Ophthalmology 2015;122:1283-93.

50. Samuelson TW, Chang DF, Marquis R, et al. A Schlemm Canal Microstent for Intraocular Pressure Reduction in Primary Open-Angle Glaucoma and Cataract: The HORIZON Study. Ophthalmology 2019;126:29-37.

51. Rehman Siddiqui MA, Khairy HA, Azuara-Blanco A. Effect of cataract extraction on SITA perimetry in patients with glaucoma. J Glaucoma 2007;16:205-8.

52. Bhandari S, Pandyal I, Khanal SP, et al. Effect of phacoemulsification surgery on various parameters in patients with glaucoma. Nepal J Ophthalmol 2014;6:46-55.

53. Altmeyer M, Wirbelauer C, Haberle H, et al. Cataract surgery in patients with end-stage glaucoma. Klin Monbl Augenheilkd 2006;223:297-302.

54. Koucheki B, Nouri-Mahdavi K, Patel G, et al. Visual field changes after cataract extraction: the AGIS experience. Am J Ophthalmol 2004;138:1022-8.

55. Musch DC, Gillespie BW, Niziol LM, et al. Cataract extraction in the collaborative initial glaucoma treatment study: incidence, risk factors, and the effect of cataract progression and extraction on clinical and quality-of-life outcomes. Arch Ophthalmol 2006;124:1694-700.

56. Chen PP, Budenz DL. The effects of cataract extraction on the visual field of eyes with chronic open-angle glaucoma. Am J Ophthalmol 1998;125:325-33.

57. Seol BR, Jeoung JW, Park KH. Changes of visual-field global indices after cataract surgery in primary open-angle glaucoma patients. Jpn J Ophthalmol 2016;60:439-45.

58. Chung HJ, Choi JH, Lee YC, et al. Effect of Cataract Opacity Type and Glaucoma Severity on Visual Field Index. Optom Vis Sci 2016;93:575-8.

59. Rao HL, Jonnadula GB, Addepalli UK, et al. Effect of cataract extraction on Visual Field Index in glaucoma. J Glaucoma 2013;22:164-8.

60. Ang GS, Shunmugam M, Azuara-Blanco A. Effect of cataract extraction on the glaucoma progression index (GPI) in glaucoma patients. J Glaucoma 2010;19:275-8.

61. Mwanza JC, Bhorade AM, Sekhon N, et al. Effect of cataract and its removal on signal strength and peripapillary retinal nerve fiber layer optical coherence 
tomography measurements. J Glaucoma 2011;20:37-43.

62. Kim NR, Lee H, Lee ES, et al. Influence of cataract on time domain and spectral domain optical coherence tomography retinal nerve fiber layer measurements. J Glaucoma 2012;21:116-22.

63. Dada T, Behera G, Agarwal A, et al. Effect of cataract surgery on retinal nerve fiber layer thickness parameters using scanning laser polarimetry (GDxVCC). Indian J Ophthalmol 2010;58:389-94.

64. Nakatani Y, Higashide T, Ohkubo S, et al. Effect of cataract and its removal on ganglion cell complex thickness and peripapillary retinal nerve fiber layer thickness measurements by fourier-domain optical coherence tomography. J Glaucoma 2013;22:447-55.

65. Jha B, Sharma R, Vanathi M, et al. Effect of phacoemulsification on measurement of retinal nerve fiber layer and optic nerve head parameters using spectral-domain-optical coherence tomography. Oman J Ophthalmol 2017;10:91-5.

66. Özyol P, Ozyol E, Sul S, et al. Intra-ocular pressure fluctuation after cataract surgery in primary angle-closure glaucoma eyes medically controlled after laser iridotomy. Acta Ophthalmol 2016;94:e528-33.

67. Xu X, Sun Q, Ma YY, et al. Vision-related Quality of Life Outcomes of Cataract Surgery in Advanced Glaucoma Patients. J Glaucoma 2016;25:e5-11.
Cite this article as: Tsui JL, Chan NC, Tham CC. The role of lens extraction in glaucoma management. Ann Transl Med 2020;8(22):1550. doi: 10.21037/atm-20-3251a 